\author{
J. H. Holstein ${ }^{1}$ - F. M. Stuby ${ }^{2}$ S. C. Herath ${ }^{1}$ U. Culemann ${ }^{3}$ - E. Aghayev ${ }^{4}$. \\ T. Pohlemann ${ }^{1}$ \\ ${ }^{1}$ Klinik für Unfall-, Hand- und Wiederherstellungschirurgie, Universitätsklinikum des Saarlandes, \\ Homburg/Saar, Deutschland \\ ${ }^{2}$ Abteilung für Unfall- und Wiederherstellungschirurgie, BG Klinik Tübingen, Tübingen, Deutschland \\ ${ }^{3}$ Klinik für Unfallchirurgie, Allgemeines Krankenhaus Celle, Celle, Deutschland \\ ${ }^{4}$ Institut für Evaluative Forschung in der Medizin, Universität Bern, Bern, Schweiz
}

\title{
Einfluss des Beckenregisters der DGU auf die Versorgung von Beckenringfrakturen
}

\section{Methoden \\ Erste Phase des Beckenregisters (AG Becken I)}

Als Folge der relativ geringen Inzidenz von Beckenringfrakturen (19 bis 37 Frakturen/100.000 Einwohner/Jahr [16]) ist die individuelle Erfahrung auch großer Traumazentren mit diesem inhomogenen Verletzungstyp limitiert. Ende der 1980er Jahre wurde daher in Deutschland die Notwendigkeit einer systematischen Datenerhebung zur Evaluation von Beckenring- (und Azetabulum-)Frakturen gesehen. Mit Gründung der AG Becken I, die von der Deutschen Sektion der AO International und der DGU finanziell unterstützt wurde, wurde am 1. Januar 1991 das Beckenregister zur Dokumentation von Beckenring- und Azetabulumfrakturen unter Teilnahme von anfänglich 10 Kliniken initiiert. Der Fokus der Dokumentation lag primär auf epidemiologischen Fragestellungen, insbesondere hinsichtlich der Inzidenz und Versorgungsrealität von Beckenring- und Azetabulumfrakturen in Deutschland. Die Datenerhebung wurde zunächst auf 2 Jahre angesetzt. Die Entwicklung einer systematischen Datenerhebung und -verarbeitung war eine der großen Herausforderungen der Initialphase des Registers. Begleitet von regelmäßigen Treffen der am Register beteiligten Arbeitsgruppen wurden die Validität und Reliabilität der Dateneingabe kontinuierlich überprüft.

Anhand der gewonnenen Daten gelang es, für die Beckenringverletzung eine besondere Verletzungsentität $\mathrm{zu}$ definieren, die bis heute mit einer überdurchschnittlich hohen Letalität vergesellschaftet ist: Der Begriff der „komplexen Beckenverletzung", die durch eine Beckenringverletzung kombiniert mit einem signifikanten Weichteilschaden charakterisiert ist und sich von der „einfachen Beckenverletzung“ abgrenzen lässt [1], hat sich als eine gängige Klassifikation auch im klinischen Alltag durchgesetzt. Darüber hinaus wurden bestehende Klassifikationen reevaluiert, um Begrifflichkeiten zur Verletzungsart klar zu definieren und voneinander abzugrenzen. Ein Beispiel hierfür sind die herausgearbeiteten Kriterien für die Unterscheidung zwischen einer ZweiPfeiler-Azetabulum- und einer Beckenringfraktur. Im Rahmen der Nachuntersuchungen spezifischer Frakturtypen wurde ein eigens entwickelter „Outcome-score" angewandt, der klinische und radiologische Ergebnisse ebenso wie die soziale Rehabilitation nach Beckenringverletzungen adressiert. Insgesamt konnten in einem Dreijahreszeitraum 1722 konsekutive Fälle in das Register eingeschlossen werden. Die Nachuntersuchung vordefinierter spezifischer Frakturtypen mindestens 2 Jahre nach Trauma umfasste 468 Patienten, was einer Rate von $73 \%$ entsprach. Die Ergebnisse dieser ersten Phase der Registerarbeit wurden in einer umfassenden Publikation zusammengefasst [13].

\section{Zweite Phase des Beckenregisters (AG Becken II)}

Die Datenanalyse der ersten Registerphase ergab, dass die Anzahl der eingeschlossenen Patienten für die Beantwortung spezifischer Fragestellungen (z. B. „Einfluss einer frühzeitigen Nervendekompression und Frakturstabilisierung auf das neurologische Patientenoutcome nach Sakrumfrakturen mit Nervenläsion") immer noch zu klein war. Entsprechend wurde das Kollektiv der am Beckenregister teilnehmenden Einrichtungen 1998 auf 22 Kliniken vergrößert. Der Basisdatensatz wurde verkleinert, um dafür verletzungsspezifische Subgruppen zu bilden, deren ergänzender Datensatz auf die Beantwortung spezifischer Fragestellungen fokussiert war. Beispielsweise sollten so in einem $\mathrm{Pa}$ tientenkollektiv mit isolierten operativ versorgten Azetabulumfrakturen prognostische Faktoren für eine posttraumatische Koxarthrose identifiziert werden. Insgesamt konnten während der zweiten Phase der Registerarbeit bis 2002 Daten von 2569 Patienten in das Register eingeschlossen werden. Die zunehmende Zahl der am Register beteiligten Einrichtungen und die wachsende Datenmenge 


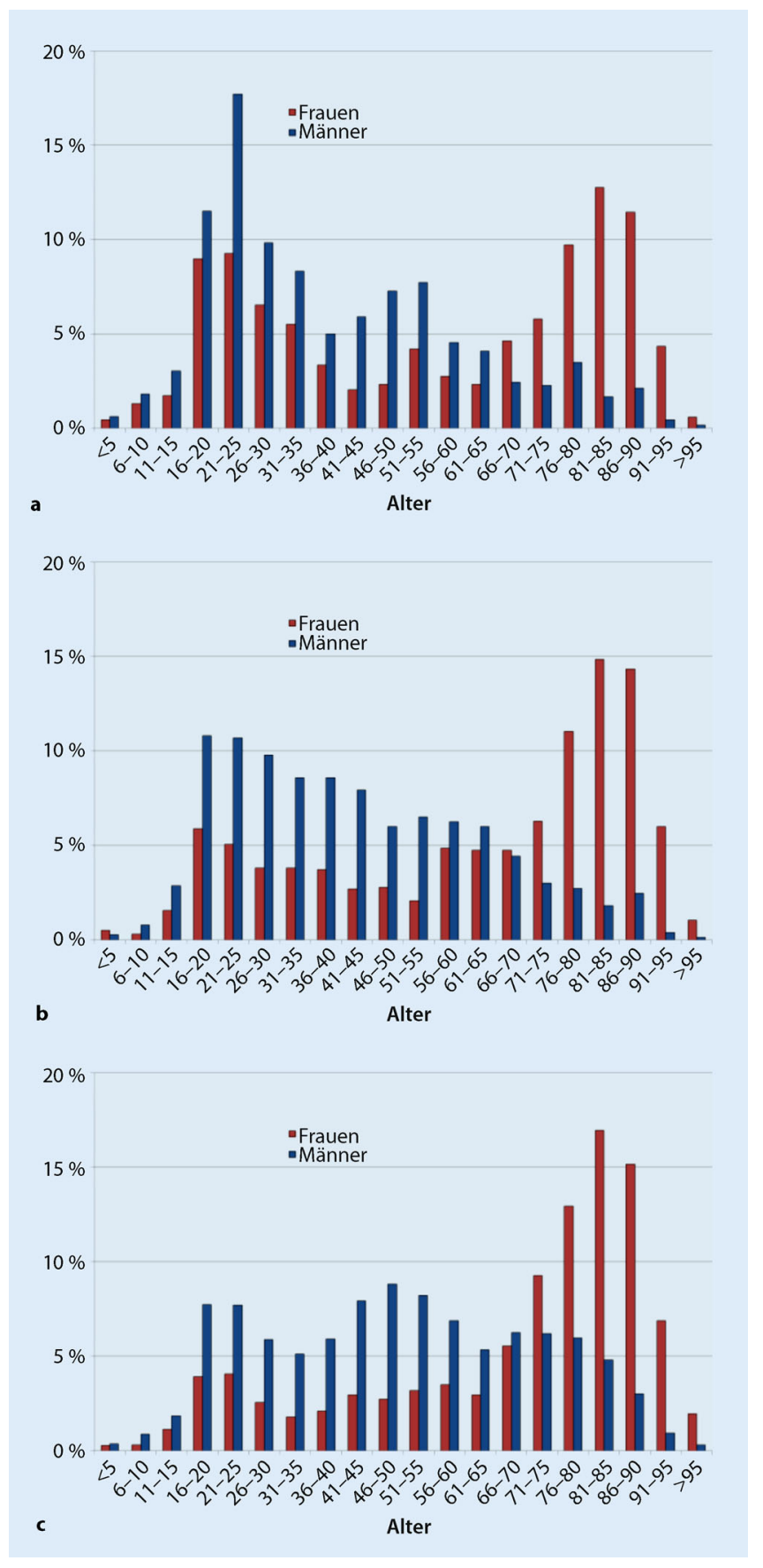

Abb. 1 A Prozentuale Altersverteilung von Beckenringfrakturen in der ersten Phase des Registers 1991-1993 (a), der zweiten Phase des Registers 1998-2002 (b) sowie der dritten Phase des Registers seit 2004 (c)

stellten eine technologische Herausforderung für die Datenakquise und -verwaltung dar, sodass die Notwendigkeit einer professionellen technologischen Plattform zur validen elektronischen Datenspeicherung evident wurde. Es bestand daher Konsens in der Arbeitsgruppe Becken, einen externen Provider

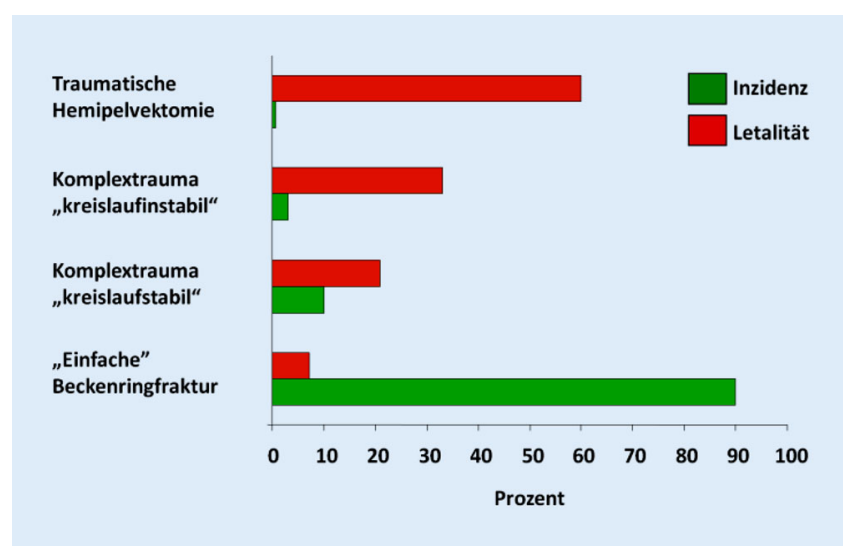

Abb. $2 \Delta$ Inzidenz und Letalität der 4 Schweregrade von Beckenringfrakturen: „einfache“ Beckenringfraktur, ,komplexe" Beckenringfraktur mit stabilem Kreislauf, "komplexe“ Beckenringfraktur mit instabilem Kreislauf, traumatische Hemipelvektomie [1]

Dokumentationsplattform MEMdoc ${ }^{\mathrm{TM}}$, betrieben durch das Institut für Evaluative Forschung in der Medizin der Universität Bern, als zentraler Provider für das Beckenregister ausgewählt. Aufgebaut auf einem vernetzten System verschiedener Technologien bot MEMdoc $^{\mathrm{TM}}$ sowohl online als auch offline Tools zur peripheren Datenerfassung und -bearbeitung. Hierbei besteht eine kontinuierliche Verbindung zwischen den beteiligen Kliniken und der zentralisierten Datenbank, über die die eingegebenen Daten unter Berücksichtigung aller Datenschutzstandards jederzeit übermittelt, verlinkt und mit einem integrierten Regelwerk validiert werden können. Das Beckenregister wurde in diesem Zuge für alle interessierten Kliniken geöffnet, insofern sie sich der kontinuierlichen und kompletten Dateneingabe verpflichteten. Erstmals nahmen somit in der Folge auch Kliniken außerhalb Deutschlands am Beckenregister teil.

\section{\) Die beteiligten Kliniken und die zentralisierte Datenbank sind kontinuierlich verbunden}

mit der elektronischen Verwaltung der Datenbank zu beauftragen.

\section{Dritte Phase des Beckenregisters (AG Becken III)}

Aufgrund seiner Expertise und Erfahrung in der Registerarbeit wurde die
Regelmäßige Meetings der am Register beteiligten Einrichtungen dienen bisheute dem engen persönlichen Austausch ebenso wie der Qualitätskontrolle der Registerarbeit. Wissenschaftlich rückten mehr und mehr spezifische Fragestellun- 


\section{J. H. Holstein - F. M. Stuby - S. C. Herath - U. Culemann - E. Aghayev - T. Pohlemann Einfluss des Beckenregisters der DGU auf die Versorgung von Beckenringfrakturen}

\section{Zusammenfassung}

Die Inzidenz von Beckenringfrakturen ist mit einem Anteil von je nach Studienlage 2-8\% aller Frakturen vergleichsweise gering. Das Erscheinungsbild einer Beckenringfraktur ist sehr vielfältig und reicht von einfachen und meist "harmlosen" Typ-A-Verletzungen bis hin zu lebensbedrohlichen komplexen Typ-C-Verletzungen. Während in der Vergangenheit postuliert wurde, dass ein hohes Energieniveau erforderlich ist, um den Beckenring zu frakturieren, wurde in den letzten Jahren nicht zuletzt durch Daten des Beckenregisters der DGU evident, dass bei osteoporotischem Knochen oft auch ein Bagatelltrauma zu einer Beckenringfraktur führen kann. Es kristallisierte sich heraus, dass bei einem rasant wachsenden geriatrischen Patientenkollektiv sogar ohne vorangegangenes Trauma Insuffizienzfrakturen des Beckenrings zu beobachten sind. Auch an großen Traumazentren ist die Anzahl der Patienten mit Beckenringfrakturen vielfach nicht ausreichend, um durch Monocenterstudien valide Aussagen über epidemiologische, diagnostische und therapeutische Entwicklungen treffen zu können. Aus diesem Grunde wurde bereits 1991 von der AG Becken der DGU das weltweit erste und bis heute einzige Register zur Dokumentation und Evaluation von Beckenverletzungen ins Leben gerufen. Standen anfänglich v. a. epidemiologische und diagnostische Fragestellungen im Vordergrund der Dokumentation, entwickelte sich im Laufe der Zeit ein zunehmend wachsender Datensatz mit umfassenden Parametern zu Verletzungsmustern, operativen und konservativen Therapieregimen sowie dem kurz- und langfristigen Outcome der Patienten. Während das Beckenregister ursprünglich in 10 Einrichtungen gestartet wurde, nehmen mittlerweile über 30 Kliniken auch außerhalb Deutschlands an der Datendokumentation teil. Allein in der dritten Phase der Registerarbeit konnten seit 2004 an die 15.000 Patienten mit Becken- und Azetabulumfrakturen in die Datenbank eingeschlossen werden. Neben dem wissenschaftlichen Einfluss des Beckenregisters, der sich in zahlreichen nationalen und internationalen Publikationen widerspiegelt, konnten durch die Datenerhebungen die sich dramatisch wandelnde Epidemiologie von Beckenringverletzungen, Weiterentwicklungen in der Diagnostik sowie die sich über den Zeitverlauf ändernden operativen Therapieverfahren aufgezeigt werden. Nicht zuletzt die aus der Datenlage abgeleiteten und heute etablierten Diagnostik- und Therapiealgorithmen bei Beckenringfrakturen spiegeln den klinischen Wert des Beckenregisters wider.

Schlüsselwörter

Register · Beckenfraktur · Epidemiologie ·

Diagnostikalgorithmen · Therapiealgorithmen

\section{Influence of the pelvic trauma registry of the DGU on treatment of pelvic ring fractures}

\section{Abstract}

Fractures of the pelvic ring are comparatively rare with an incidence of $2-8 \%$ of all fractures depending on the study in question. The severity of pelvic ring fractures can be very different ranging from simple and mostly "harmless" type A fractures up to life-threatening complex type $C$ fractures. Although it was previously postulated that high-energy trauma was necessary to induce a pelvic ring fracture, over the past decades it became more and more evident, not least from data in the pelvic trauma registry of the German Society for Trauma Surgery (DGU), that low-energy minor trauma can also cause pelvic ring fractures of osteoporotic bone and in a rapidly increasing population of geriatric patients insufficiency fractures of the pelvic ring are nowadays observed with no preceding trauma.

Even in large trauma centers the number of patients with pelvic ring fractures is mostly insufficient to perform valid and sufficiently powerful monocentric studies on epidemiological, diagnostic or therapeutic issues. For this reason, in 1991 the first and still the only registry worldwide for the documentation and evaluation of pelvic ring fractures was introduced by the Working Group Pelvis (AG Becken) of the DGU. Originally, the main objectives of the documentation were epidemiological and diagnostic issues; however, in the course of time it developed into an increasingly expanding dataset with comprehensive parameters on injury patterns, operative and conservative therapy regimens and shortterm and long-term outcome of patients. Originally starting with 10 institutions, in the meantime more than 30 hospitals in Germany and other European countries participate in the documentation of data. In the third phase of the registry alone, which was started in 2004, data from approximately 15,000 patients with pelvic ring and acetabular fractures were documented. In addition to the scientific impact of the pelvic trauma registry, which is reflected in the numerous national and international publications, the dramatically changing epidemiology of pelvic ring fractures, further developments in diagnostics and the changes in operative procedures over time could be demonstrated. Last but not least the now well-established diagnostic and therapeutic algorithms for pelvic ring fractures, which could be derived from the information collated in registry studies, reflect the clinical impact of the registry.

Keywords Registry · Pelvic fracture - Epidemiology . Diagnostics algorithms - Therapy algorithms 


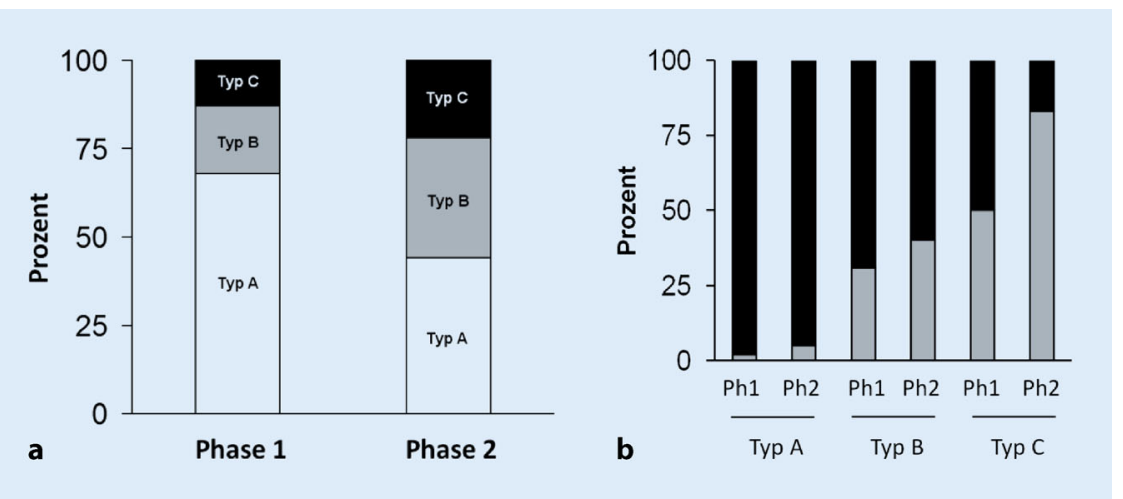

Abb. $3 \Delta$ a Prozentuale Verteilung der Frakturtypen nach Tile [18] sowie b prozentualer Anteil der operativen ( $\mathrm{grau}$ ) vs. konservativen (schwarz) Versorgung des jeweiligen Frakturtyps in der ersten (1991-1993) und zweiten (1998-2002) Phase des Registers [19]. Ph Phase

gen in den Fokus der Datenauswertung. Neben Untersuchungen $\mathrm{zu}$ Therapiekonzepten und epidemiologischen Entwicklungen konnten beispielsweise auch Mortalitätsprädiktoren und Einflussfaktoren auf die Lebensqualität bei Patienten mit Beckenringfrakturen herausgearbeitet werden $[5,9,10]$. Bis dato konnten in der dritten Phase des Registers seit 2004 an die 15.000 Patienten aus 3 europäischen Ländern (Deutschland, Belgien, Schweden) eingeschlossen werden.

\section{Ergebnisse}

\section{Erste Phase des Beckenregisters (AG Becken I)}

Die epidemiologische Auswertung der Registerdaten von 1991-1993 ergab für die Inzidenz von Beckenringfrakturen eine dreigipflige Altersverteilung (• Abb. 1). Assoziiert mit Hochrasanztraumata war das größte Patientenkollektiv unter Männern in der 3. Lebensdekade $\mathrm{zu}$ finden. Interessanterweise war aber schon zu diesem frühen Zeitpunkt der Registerarbeit ein zweiter großer Peak im Kollektiv der geriatrischen weiblichen Patienten auffällig (• Abb. 1).

Hinsichtlich des Verletzungsmusters konnten 4 gestaffelte Schwergrade der Beckenringfraktur definiert werden, anhand derer die Mortalität der Patienten prognostisch abgeschätzt werden kann. Unterschieden wurden die „einfache“ Beckenringfraktur, die „komplexe“ Beckenringfraktur mit stabilem Kreislauf, die „komplexe“ Beckenringfraktur mit instabilem Kreislauf sowie die traumati- sche Hemipelvektomie (• Abb. 2). Für die 4 Schweregrade der Beckenringfraktur zeigte sich eine inverse Relation zwischen Inzidenz und Mortalität (• Abb. 2; [1]).

Die klinischen Nachuntersuchungen ergaben $u$. a. persistierende Schmerzen abhängig vom Frakturtyp bei bis zu 73 \% der Patienten und anhaltende urogenitale Symptome bei bis $\mathrm{zu} 12 \%$ der Patienten [15]. Ein anatomisches Ausheilungsergebnis konnte radiologisch hingegen in $75 \%$ der Typ-C- (Tile-Klassifikation [18]) und in über $90 \%$ der Typ-BVerletzungen nachgewiesen werden [13]. Insbesondere die klinischen Ergebnisse zeigten zu diesem Zeitpunkt ein nicht zufriedenstellendes Outcome nach Beckenringfrakturen, sodass eine Reevaluation und Optimierung der gängigen Therapiekonzepte erforderlich schien.

\section{Zweite Phase des Beckenregisters (AG Becken II)} deutete sich eine rasante Zunahme von Beckenringfrakturen bei geriatrischen Patienten an. So lag der größte Peak in der Altersverteilung anders als noch in der ersten Phase des Registers nicht mehr bei jungen männlichen Patienten, sondern bei weiblichen geriatrischen Patienten in der 9. Lebensdekade (• Abb. 1). Aber nicht nur in der Altersverteilung, auch im Verhältnis der Frakturtypen zeigte sich ein deutlicher Wandel. So nahm die Inzidenz von Typ-A-Verletzungen im Vergleich zur ersten Registerphase $a b$, während vermehrt Typ-B- und -C-Verletzungen nachgewiesen wurden
In der zweiten Phase der Registerarbeit
(- Abb. 3; [19]). Genauere Analysen ergaben, dass Fortschritte in der Diagnostik und hierbei insbesondere eine großzügigere Anwendung von Schichtbildverfahren nativradiologisch initial unerkannte Frakturen des hinteren Beckenrings gerade bei älteren Patienten aufdeckten [20]. Therapeutisch fiel eine Zunahme der operativen Versorgung von Beckenringfrakturen v. a. bei TypC-Verletzungen auf (• Abb. 3; [19]).

\section{Dritte Phase des Beckenregisters (AG Becken III)}

Epidemiologische Entwicklungstrends, die sich bereits in der zweiten Phase des Registers abzeichneten, setzten sich in der dritten Phase in beachtlicherweise Weise fort. So ist nach aktueller Datenlage der erste Alterspeak junger Patienten nahezu vollständig nivelliert, während die Inzidenz von Beckenringfrakturen bei den geriatrischen weiblichen Patienten weiter zugenommen hat (• Abb. 2). Dieser Wandel der Beckenringfraktur von einer Hochrasanzverletzung hin $\mathrm{zu}$ einer Altersfraktur hat natürlich weitreichende Auswirkungen auf die Versorgungsrealität der Beckenringfraktur, die Gegenstand einer aktuellen Auswertung der Registerdaten ist.

\section{》) Epidemiologische \\ Entwicklungstrends der zweiten setzten sich in der dritten Phase fort}

In weiteren spezifischen Untersuchungen wurde die Wertigkeit der verschiedenen Maßnahmen in der Notfallbehandlung von Beckenringfrakturen analysiert [8, 12]. Methodisch gelang es, den Datensatz des Beckenregisters mit dem Datensatz des TraumaRegisters DGU ${ }^{\circledR}$ zu matchen [3]. Entwicklungen wie die Einführung der präklinischen externen Notfallstabilisierung von Beckenringfrakturen, aber auch Fragestellungen zur Volumentherapie und Massentransfusion bei Patienten mit komplexen Beckenverletzungen konnten so reflektiert werden $[2,5]$.

Neben der Notfalltherapie lag der Fokus anderer Studien auf Trends in 


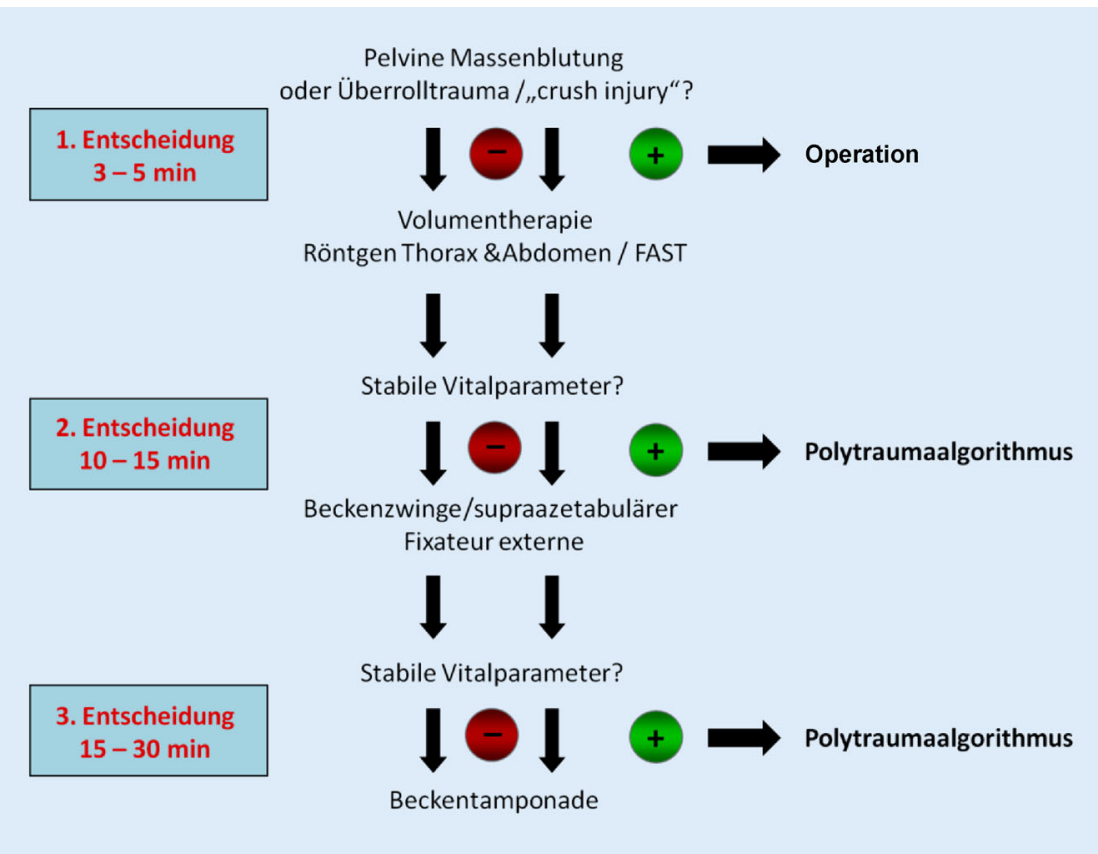

Abb. 4 \ Therapiealgorithmus für die Notfallbehandlung komplexer Beckenverletzungen. (Adaptiert nach [21], mit freundl. Genehmigung von Elsevier). FAST Focused Assessment with Sonography for Trauma

der definitiven Versorgung von Beckenringfrakturen. Beispielhaft sei in diesem Zusammenhang eine vergleichende Auswertung navigierter und konventioneller Osteosyntheseverfahren am hinteren Beckenring genannt [22]. Große Outcomeanalysen adressierten neben Komplikationen und Mortalität erstmals systematisch an einem großen Patientenkollektiv Einflussfaktoren auf die Lebensqualität nach Beckenringfraktur $[10,14,17]$.

\section{Diskussion}

Register zur klinischen Datenerhebung sind heute anerkannte und weitverbreitete Werkzeuge der Qualitätssicherung und wissenschaftlichen Arbeit. Insbesondere für Krankheiten, Pathologien oder Verletzungen mit niedriger Inzidenz ist ein Register eine nahezu alternativlose Methode, um große Datenmengen zu akquirieren, die valide Rückschlüsse auf Epidemiologie, Versorgungsstrukturen und Behandlungsoutcome der betroffenen Patienten zulassen. Vor diesem Hintergrund wurde bereits 1991 von der AG Becken der DGU das Beckenregister zur Dokumentation von Beckenringund Azetabulumfrakturen ins Leben ge- rufen. Bis zum heutigen Tage gelang es in 3 Phasen, annähernd 20.000 Patienten in das Register einzuschließen. Aus diesem Datensatz konnten über die Jahre zahlreiche Informationen insbesondere über zeitliche Entwicklungen und Veränderungen in der Versorgungsrealität von Beckenring- (und Azetabulum)Frakturen gewonnen werden. Dieses Wissen, das heute of als selbstverständlich hingenommen wird, spiegelt sich in den aktuellen Diagnosealgorithmen ebenso wie in den verschiedenen Neuund Weiterentwicklungen von Behandlungsmaßnahmen der Notfall- und Elektivversorgung von Beckenringfrakturen wider.

Als Beispiel sei in diesem Zusammenhang der große Themenkomplex der Altersfrakturen genannt. Herrschte lange Zeit die Meinung vor, eine Beckenringverletzung könne nur durch ein hohes Energieniveau verursacht werden, zeigten bereits die Daten der ersten Registerphase, dass ein Großteil der Beckenringfrakturen in einem geriatrischen Patientenkollektiv $\mathrm{zu}$ finden ist, das eben nicht einem Hochrasanztrauma ausgesetzt war. Analysen der Registerdaten brachten zu Tage, dass diese initial meist als harmlos und sta- bil eingeschätzten Typ-A-Verletzungen häufig mit persistierenden Beschwerden der Patienten vergesellschaftet waren. Weitere Untersuchungen ergaben, dass eine Ursache hierfür in einer Fehleinschätzung des Frakturtyps lag, sodass viele Typ-B-Verletzungen aufgrund unzureichender Diagnostik als Typ-AVerletzung missinterpretiert wurden. In Konsequenz dieser Erkenntnisse wurden Algorithmen definiert, die u. a. durch eine angepasste Bildgebung zu einer sensitiveren Diagnostik und strukturierten Behandlung von Beckenringfrakturen im Alter führten $[6,19,20]$.

\section{》) Bei Altersfrakturen wurde der Frakturtyp oft falsch eingeschätzt}

Die Notwendigkeit modifizierter Osteosynthesetechniken bei geriatrischen Patienten verglichen zu den herkömmlichen Verfahren bei jungen Patienten wurde durch die Datenlage zunehmend evident [7]. Die Tatsache, dass entsprechend der vorliegenden Registerdaten das Patientenalter der stärkste Prädiktor für die Lebensqualität nach einer Beckenringfraktur ist, offenbart die große Herausforderung einer Verbesserung der Versorgungsprinzipien geriatrischer Patienten mit Beckenringfraktur [10].

Als weiteres Beispiel für den klinischen Einfluss der Registerarbeit seien die gewonnen Erkenntnisse über das Notfallmanagement von Beckenringfrakturen erwähnt. Durch die Begrifflichkeit der „komplexen Beckenverletzung“, die durch eine Beckenringfraktur mit signifikantem Weichteilschaden gekennzeichnet ist, konnte eine besondere Verletzungsentität definiert werden, die sich auch in Folgestudien als Indikator für eine besonders hohe Mortalität erwies [1, $4,9]$. Gewonnene Erkenntnisse fanden durch Entwicklung eines eigenen Therapiealgorithmus für die Notfallbehandlung komplexer Beckenverletzungen Eingang in den klinischen Alltag (• Abb.4; [21]). Die Effektivität heute als nahe$\mathrm{zu}$ selbstverständlich angewandter $\mathrm{Me}$ thoden zur Notfallstabilisierung instabiler Beckenringverletzungen, wie etwa des „pelvic binder“, konnten durch Aus- 
wertung der Registerdaten nachgewiesen werden [12].

Neben dem wissenschaftlichen Wert als Instrument der Versorgungsforschung können Register auch als wertvolle Quelle der externen Qualitätskontrolle herangezogen werden [11]. Prinzipiell kann jede am Beckenregister teilnehmende Einrichtung über den zentralen Provider die gespeicherten Registerdaten anfordern. Hierdurch können die Institutionen deskriptive Informationen über die Versorgungsrealität von Beckenfrakturen in der eigenen Klinik, aber auch einen Abgleich über die Leistungsfähigkeit der eigenen Klinik im Verhältnis zu den anderen Registerteilnehmern abrufen. Insbesondere klinikinterne Entwicklungen und Veränderungen beispielsweise in angewandten Operationsverfahren oder auch in der Patientenmortalität können somit über definierte Zeiträume reflektiert und analysiert werden.

\section{Fazit für die Praxis}

\section{- Das Beckenregister ist ein wichtiges Instrument zur Abbildung der Versor- gungsrealität von Beckenfrakturen in Deutschland und zunehmend auch anderen teilnehmenden Ländern. \\ - Insbesondere die epidemiologischen Entwicklungen, aber auch diagnos- tische und therapeutische Trends werden durch das Register besonders aussagekräftig widergegeben. - Darüber hinaus dienen die erhobe- nen Daten den beteiligten Kliniken als wichtiges Werkzeug der externen Qualitätskontrolle.}

\section{Korrespondenzadresse}

\section{PD Dr. J. H. Holstein}

Klinik für Unfall-, Hand- und Wiederherstellungschirurgie, Universitätsklinikum des Saarlandes

Kirrbergerstraße 100, 66421 Homburg/Saar, Deutschland

joerg.holstein@uks.eu

Danksagung. Wir danken allen Mitgliedern der AG Becken, ohne deren großes Engagement das Beckenregister nicht möglich wäre. Teilnehmende Kliniken sind: AKH Celle, AZ Groeninge, AZ St Dimpa Geel, BG Unfallklinik Bergmannstrost Halle, BG Unfallklinik Frankfurt/Main, BG Unfallklinik
Ludwigshafen, BG Unfallklinik Murnau, BG Unfallklinik Tübingen, BWK Ulm, Centre Hospitalier Jolimont-Lobbes, Charité Berlin,

Diakonissenkrankenhaus Flensburg, Friederikenstift Hannover, Hospital Universitaire Saint Luc Brüssel, Klinikum Augsburg, Klinikum Dortmund, Klinikum Nürnberg, Klinikum Ludwigsburg, Klinikum rechts der Isar München, Klinikum Schwerin, Kreisklinik Biberach, Medizinische Hochschule Hannover, Sahlgrenska Universitetssjukhuset Göteborg, SKM Koblenz, Städtisches Klinikum Braunschweig, Städtisches Klinikum Karlsruhe, STER Antwerpen, Universitätsklinikum Aachen, Universitätsklinikum des Saarlandes, Universitätsklinikum Freiburg, Universitätsklinikum Halle, Universitätsklinikum Hamburg-Eppendorf, Universitätsklinikum Jena, Universitätsklinikum Kiel, Universitätsklinikum Leipzig, Universitätsklinikum Magdeburg, Universitätsklinikum Mainz, Universitätsklinikum Münster, Universitätsklinikum Regensburg, Universitätsklinikum Ulm, Westpfalz-Klinikum Kaiserslautern. Wir danken zudem Frau S. Drum, Studienschwester an Klinik für Unfall-, Hand- und Wiederherstellungschirurgie, Universitätsklinikum des Saarlandes, für die großartige Unterstützung bei der Datenauswertung.

\section{Einhaltung ethischer Richtlinien}

Interessenkonflikt. J. H. Holstein, F.M. Stuby, S.C. Herath, U. Culemann und T. Pohlemann weisen auf folgende Beziehungen ihn: J. H. Holstein, F.M. Stuby, S.C. Herath, U. Culemann und T. Pohlemann sind an den oben genannten Kliniken tätig. F.M. Stuby ist zudem Leiter der AG Becken, J.H. Holstein ist stellvertretender Leiter der AG Becken. E. Aghayev weist auf folgende Beziehung hin: E. Aghayev ist am Institut für Evaluative Forschung in der Medizin der Universität Bern beschäftigt, das die Dokumentationsplattform MEMdoc $^{\mathrm{TM}}$ betreibt.

Dieser Beitrag beinhaltet keine von den Autoren durchgeführten Studien an Menschen oder Tieren.

\section{Literatur}

1. Bosch U, Pohlemann T, Haas N et al (1992) Classification and management of complex pelvic trauma. Unfallchirurg 95:189-196

2. Burkhardt M, Kristen A, Culemann U et al (2014) Pelvic fracture in multiple trauma: are we still upto-date with massive fluid resuscitation? Injury 45(Suppl 3):70-75

3. Burkhardt M, Nienaber U, Holstein JH et al (2013) Trauma registry record linkage: methodological approach to benefit from complementary data using the example of the German Pelvic Injury Register and the TraumaRegister DGU(R). BMC Med Res Methodol 13:30

4. Burkhardt M, Nienaber U, Krause J et al (2015) Complex pelvic traumas: Data linkage of the German Pelvic Injury Register and the TraumaRegister DGU(R). Unfallchirurg 118:957-962

5. Burkhardt M, Nienaber U, Pizanis A et al (2012) Acute management and outcome of multiple trauma patients with pelvic disruptions. Crit Care 16:R163

6. Culemann U, Scola A, Tosounidis G et al (2010) Concept for treatment of pelvic ring injuries in elderly patients. A challenge. Unfallchirurg 113:258-271

7. Fuchs T, Rottbeck U, Hofbauer V et al (2011) Pelvic ring fractures in the elderly. Underestimated osteoporotic fracture. Unfallchirurg 114:663-670

8. Hauschild O, Aghayev E, Heyden J Von et al (2012) Angioembolization for pelvic hemorrhage control: results from the German pelvic injury register. JTrauma Acute Care Surg 73:679-684

9. Holstein JH, Culemann U, Pohlemann T (2012) What are predictors of mortality in patients with pelvic fractures? Clin Orthop Relat Res 470:2090-2097

10. Holstein JH, Pizanis A, Kohler D et al (2013) What are predictors for patients' quality of life after pelvic ring fractures? Clin Orthop Relat Res 471:2841-2845

11. Lefering R, Paffrath T, Nienaber U (2014) The TraumaRegister DGU(R) as data source for monitoring severe injuries. Bundesgesundheitsblatt Gesundheitsforschung Gesundheitsschutz 57:660-665

12. Pizanis A, Pohlemann T, Burkhardt M et al (2013) Emergency stabilization of the pelvic ring: Clinical comparison between three different techniques. Injury 44:1760-1764

13. Pohlemann T, Gänsslen A, Hartung S (1997) Results of a german multicentre study group. Springer, Heidelberg

14. Pohlemann T, Stengel D, Tosounidis $G$ et al (2011) Survival trends and predictors of mortality in severe pelvic trauma: estimates from the german pelvic trauma registry initiative. Injury 42:997-1002

15. Pohlemann T, Tscherne $H$, Baumgartel $F$ et al (1996) Pelvic fractures: epidemiology, therapy and long-term outcome. overview of the multicenter study of the pelvis study group. Unfallchirurg 99:160-167

16. Ragnarsson B, Jacobsson B (1992) Epidemiology of pelvic fractures in a Swedish county. Acta Orthop Scand 63:297-300

17. Schmal H, Hauschild O, Culemann U et al (2010) Identification of riskfactorsforneurological deficits in patients with pelvic fractures. Orthopedics. doi:10.3928/01477447-20100625-13

18. Tile M (1996) Acutepelvicfractures:I.causation and classification. J Am Acad Orthop Surg 4:143-151

19. Tosounidis G, Holstein JH, Culemann U et al (2010) Changes in epidemiology and treatment of pelvic ring fractures in germany: an analysis on data of german pelvic Multicenter study groups I and III (DGU/AO). Acta Chir Orthop Traumatol Cech 77:450-456

20. Tosounidis G, Wirbel R, Culemann U et al (2006) Misinterpretation of anterior pelvic ring fractures in the elderly. Unfallchirurg 109:678-680

21. Tscherne H, Pohlemann T (1998) Becken und Acetabulum. Springer, Berlin

22. Zwingmann J, Sudkamp NP, Konig B et al (2013) Intra- and postoperative complications of navigated and conventional techniques in percutaneous iliosacral screw fixation after pelvic fractures: results from the german pelvic trauma registry. Injury 44:1765-1772 